\title{
Electronic states in a graphene flake strained by a Gaussian bump
}

\author{
D. Moldovan, ${ }^{*}$ M. Ramezani Masir, ${ }^{\dagger}$ and F. M. Peeters ${ }^{\ddagger}$ \\ Departement Fysica, Universiteit Antwerpen Groenenborgerlaan 171, B-2020 Antwerpen, Belgium
}

(Received 29 March 2013; revised manuscript received 3 June 2013; published 30 July 2013)

\begin{abstract}
The effect of strain in graphene is usually modeled by a pseudomagnetic vector potential which is, however, derived in the limit of small strain. In realistic cases deviations are expected in view of graphene's very high strain tolerance, which can be up to $25 \%$. Here we investigate the pseudomagnetic field generated by a Gaussian bump and we show that it exhibits significant differences with numerical tight-binding results. Furthermore, we calculate the electronic states in the strained region for a hexagon shaped flake with armchair edges. We find that the sixfold symmetry of the wave functions inside the Gaussian bump is directly related to the different effects of strain along the fundamental directions of graphene: zigzag and armchair. Low energy electrons are strongly confined in the armchair directions and are localized on the carbon atoms of a single sublattice.
\end{abstract}

DOI: 10.1103/PhysRevB.88.035446

PACS number(s): 73.22.Pr, 62.20.-x, 71.70.Di

\section{INTRODUCTION}

A single layer of carbon atoms called graphene has become a very active field of research in nanophysics. ${ }^{1,2}$ Graphene has excellent electrical and thermal properties, e.g., massless and chiral Dirac fermions which move with a Fermi velocity of about $1 / 300$ the speed of light, a linear spectrum close to the $K$ and $K^{\prime}$ points ${ }^{1,3}$ of the Brillouin zone, an anomalous integer quantum Hall effect in the presence of a magnetic field, the Klein paradox, i.e., unusual high transmission when electrons pass classically forbidden regions, the Aharonov-Bohm effect in graphene rings, extraordinary stiffness, unexpected mechanical properties, and thermomechanical and electronic properties that are highly affected by external particles and dopants. These properties of graphene have attracted considerable attention and make it a promising material for future electronic and optoelectronic devices.

An interesting recent prediction is that a geometrical deformation of the graphene lattice results in local strain that acts as a pseudomagnetic field on the electronic degrees of freedom and which leads to a pseudo-quantum-Hall effect. ${ }^{4}$ Deformation due to elastic strain changes the hopping amplitude of the carbon atoms and induces an effective vector potential that shifts the Dirac points. ${ }^{5}$ With a proper geometrical deformation it is possible to create large pseudomagnetic fields which can reach up to several hundreds of tesla. ${ }^{6,7}$ Over the past few years much effort has been devoted to finding ways of controlling graphene's electronic properties by strain. Applying in-plane strain with triangular symmetry has been shown ${ }^{4}$ theoretically to result in an uniform pseudomagnetic field of the order of $10 \mathrm{~T}$. It was reported experimentally ${ }^{8}$ that nanobubbles grown on a $\mathrm{Pt}(111)$ surface induce pseudomagnetic fields of more than $300 \mathrm{~T}$. Landau quantization of the electronic spectrum was observed by scanning tunneling microscopy. Thus, with such large strain-induced pseudomagnetic fields, one can control the electronic properties of graphene through strain engineering. ${ }^{9}$ Recently, it was shown experimentally that an external nonuniform electric field is able to induce local deformations in graphene with different curved shapes ${ }^{10}$ and thus one should be able to induce a pseudomagnetic field through an electric field.

In this paper we investigate the different effects that are induced by inhomogeneous strain in graphene. We consider a hexagon shaped graphene flake that is strained out of plane by a Gaussian bump placed in its center. The effects of strain in graphene can be modeled using a pseudomagnetic vector potential. In the case of a Gaussian bump, the traditional form of this vector potential ${ }^{11}$ results in a threefold symmetric pseudomagnetic field, as illustrated in Fig. 1(b). Recently, it has been shown in Ref. 12 that additional lattice corrections are required in order to accurately calculate the pseudomagnetic vector potential. However, these strain-induced lattice vector corrections do not contribute to the pseudomagnetic field and may be neglected. ${ }^{13-15}$ Only the strain-induced hopping parameter changes will affect the intensity of the pseudomagnetic field, but this is generally derived only up to first order in strain. Given graphene's excellent mechanical properties, it can sustain strain up to $25 \% .{ }^{16}$ At that point strain can no longer be considered to be small. For this reason, we investigate additional corrections to the vector potential to higher order in the strain and we compare this pseudomagnetic field model to results obtained with the full tight-binding result.

Furthermore, we investigate the confinement of electrons inside the strained region. It was shown earlier, using the Dirac equation formalism, that such a Gaussian bump results in low energy localized states. ${ }^{17,18}$ However, those models do not fully explain the origin of the sixfold symmetry of the localized states. Here we investigate the system using the tight-binding model and show that the influence of strain in the zigzag (zz) and armchair (ac) directions of graphene result in different pseudomagnetic fields and consequently to different localization properties for the electrons. Furthermore, we examine the energy levels and wave functions in order to show the different confinement regimes.

This paper is organized as follows. In Sec. II we present the tight-binding model, the system geometry, and the specific strain model that we use in the present paper. In Sec. III we evaluate the different approximations for the pseudomagnetic field for high strain. In Sec. IV we calculate the electronic states using the tight-binding approach and we compare the results with the Landau levels predicted by the pseudomagnetic field model. We also examine the confined electronic states inside the strained region. Our concluding remarks are given in Sec. V. 

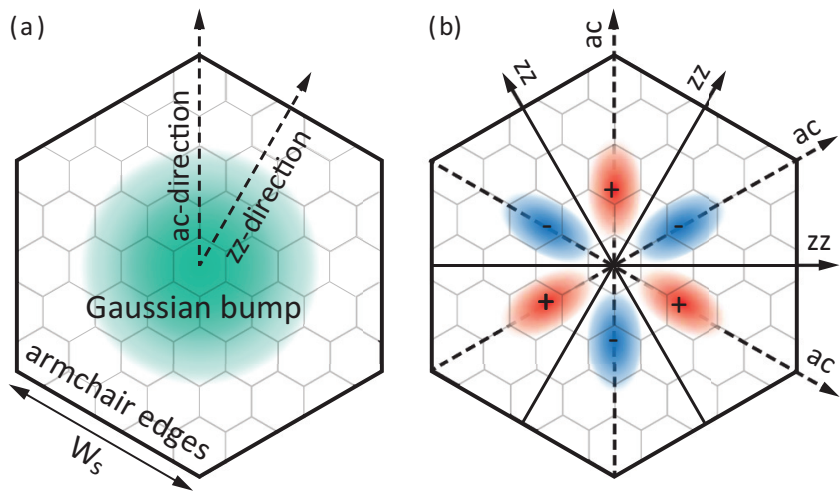

FIG. 1. (Color online) (a) Hexagon shaped graphene flake with armchair edges strained by a Gaussian bump in the center. The dashed arrows show the armchair and zigzag directions in the radial direction of the bump. (b) The bump-generated pseudomagnetic field, as calculated from the traditional form of the pseudovector potential. Red (blue) color corresponds to positive (negative) magnetic field.

\section{THE MODEL}

We consider the tight-binding model of graphene with the nearest-neighbor Hamiltonian,

$$
H=\sum_{m, n} t_{m n} a_{m}^{\dagger} b_{n}+\text { H.c. }
$$

Here $t_{m n}$ is the strained hopping energy between nearestneighbor atoms at lattice positions $m$ and $n$, while $a_{m}$ and $b_{n}$ are field operators acting, respectively, on sublattices $A$ and $B$ at their given positions. Previously, it has been shown that the strained hopping parameter is given by ${ }^{19}$

$$
t_{m n}=t_{0} e^{-\beta \omega_{m n}},
$$

where $\omega_{m n}=l_{m n} / a_{c c}-1$. Here $t_{0}=-2.8 \mathrm{eV}$ is the unstrained hopping parameter, $l_{m n}$ is the strained distance between atoms $m$ and $n, a_{c c}=0.142 \mathrm{~nm}$ is the unstrained carbon-carbon distance, and $\beta=3.37$ is the strained hopping energy modulation factor. The nearest-neighbor vectors are $\vec{d}_{1}=a_{c c}(0,1)$, $\vec{d}_{2}=a_{c c} / 2(\sqrt{3},-1)$, and $\vec{d}_{3}=a_{c c} / 2(-\sqrt{3},-1)$, as shown in Fig. 2(a). The corresponding Brillouin zone and the six $K$ points are shown in Fig. 2(b).
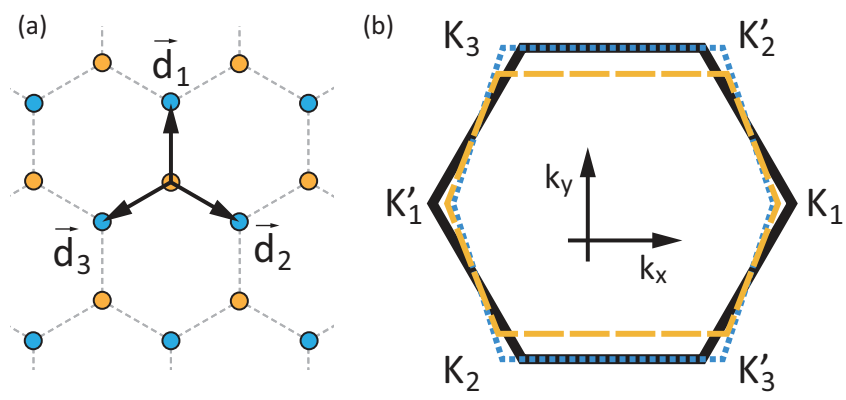

FIG. 2. (Color online) (a) The unstrained nearest-neighbor vectors $\vec{d}_{n 0}$. (b) The six $K$ points in the unstrained Brillouin zone (black, solid). The zone is also shown for $20 \%$ armchair uniaxial strain, as calculated from the first approximation of the pseudomagnetic vector potential (blue, dotted) and from the full solution of the vector potential (orange, dashed).
In the present paper we consider a finite size system which is taken as a hexagon with armchair edges. There are $N_{S}$ atoms on the hexagon edge, which corresponds to an edge width of $W_{S}=a_{c c}\left(3 N_{S} / 2-1\right)$. The total number of atoms in this hexagonal system is $N=9 N_{S}\left(N_{S} / 2-1\right)+6$. We limit ourselves to a hexagonal system that consists only of armchair edges in order to avoid the presence of zigzag edge states which would draw attention away from the bump-induced states. In the following calculations we take an edge width of $W_{S}=9 \mathrm{~nm}$, which corresponds with a flake consisting of $8322 \mathrm{C}$ atoms. The $x$ axis of the system is aligned with the zigzag direction in graphene.

In our model we strain the graphene flake by using a Gaussian bump located at the center of this system, as illustrated in Fig. 1(a). Such a strain profile can be induced with a scanning tunneling microscope (STM) tip. ${ }^{20}$ The bump's height profile is given by $h(r)=h_{0} e^{-r^{2} / b^{2}}$, where $r$ is the distance from the center of the system, and $h_{0}$ and $b$ are parameters that characterize the Gaussian bump. The Gaussian function is defined to infinity $(r \rightarrow \infty)$, which is inconvenient because increasing the system size would also change the total area of the bump. For that reason we add a cutoff radius $R$ after which the height of the bump will be zero. With this cutoff the bump height profile is expressed as

$$
h(r)=h_{0} e^{-r^{2} / b^{2}} \Theta(R-r),
$$

where $\Theta$ is the Heaviside step function. It is important to choose the cutoff radius $R$ correctly in relation to the width parameter $b$ so that the most significant part of the bump is included before the cutoff. Taking $R=3 b / \sqrt{2}$ will ensure that $99.7 \%$ of the Gaussian is inside the radius $R$. In the following calculations we take $R=6.2 \mathrm{~nm}$ as typically realized in experiments. ${ }^{8}$

\section{THE PSEUDOMAGNETIC FIELD}

The pseudomagnetic vector potential in graphene $\vec{A}_{p s}=$ $\left(\operatorname{Re} A_{p s}, \operatorname{Im} A_{p s}\right)$ is given by ${ }^{6}$

$$
A_{p s}=\frac{1}{e v_{F}} \sum_{n=1}^{3} t_{n} e^{-i \vec{K} \cdot \vec{d}_{n}},
$$

where $\vec{d}_{n}$ and $t_{n}$ are the strained nearest-neighbor vectors and hopping parameters, respectively, and $\vec{K}$ is the location of a $K$ point. The pseudomagnetic field is found as $\vec{B}_{p s}=\vec{\nabla} \times \vec{A}_{p s}$.

The strained hopping parameter from Eq. (2) can be expanded to third order as

$$
\begin{gathered}
t_{n} \approx t_{0}+\delta t_{n}^{(1)}+\delta t_{n}^{(2)}+\delta t_{n}^{(3)}, \\
t_{n} \approx t_{0}\left(1-\beta \omega_{n}+\frac{1}{2} \beta^{2} \omega_{n}^{2}-\frac{1}{6} \beta^{3} \omega_{n}^{3}\right) .
\end{gathered}
$$

The nearest-neighbor vectors $\vec{d}_{n}$ are also strained, but their total contribution to the pseudomagnetic field is zero for any strain, so they may be safely neglected. ${ }^{13,14}$ While their inclusion would change the value of the vector potential, the resulting field would not be affected. As we are mainly interested in the pseudomagnetic field, we will use the unstrained values of the vectors which are constant. Because of the out-of-plane deformation, the hopping will also be affected by curvature 
(hybridization between $\pi$ and $\sigma$ bands), but this contribution may be omitted as it is 100-1000 times smaller than the changes induced by the bond length modulation. ${ }^{6}$

Plugging the expansion (5) into Eq. (4), we can expand the pseudomagnetic vector potential to third order as

$$
A_{p s} \approx \frac{1}{e v_{F}} \sum_{n=1}^{3}(\underbrace{\delta t_{n}^{(1)}}_{A_{1}}+\underbrace{\delta t_{n}^{(2)}}_{A_{2}}+\underbrace{\delta t_{n}^{(3)}}_{A_{3}}) e^{-i \vec{K} \cdot \vec{d}_{n}},
$$

which we subdivided into three parts $A_{i} . A_{1}$ is a first order term that was originally derived in Ref. 11. $A_{2}$ and $A_{3}$ are second and third order terms which turn out to be important for large strain.

Figure 3(a) shows the pseudomagnetic field calculated from the first approximation $\left(A_{1}\right)$ of the vector potential. It exhibits threefold symmetry with positive and negative peaks along the armchair directions of graphene and zero field along the zigzag directions. The pseudomagnetic field based on the full vector potential, Eq. (4), without any approximations, is shown in Fig. 3(b). To better see the difference in field magnitude between the different approximations, we take a cut along the armchair direction of graphene, as show in Fig. 3(c). We compare the pseudomagnetic field resulting from the vector potential approximations with successively higher terms included $\left(A_{1}, A_{2}\right.$, and $\left.A_{3}\right)$ with the full form $A_{\text {full }}$ from Eq. (4). The differences are shown in Fig. 3(d) as $A_{\text {diff }}^{(i)}=$ $A_{i}-A_{\text {full }}$. The first order approximation $A_{1}$ overestimates
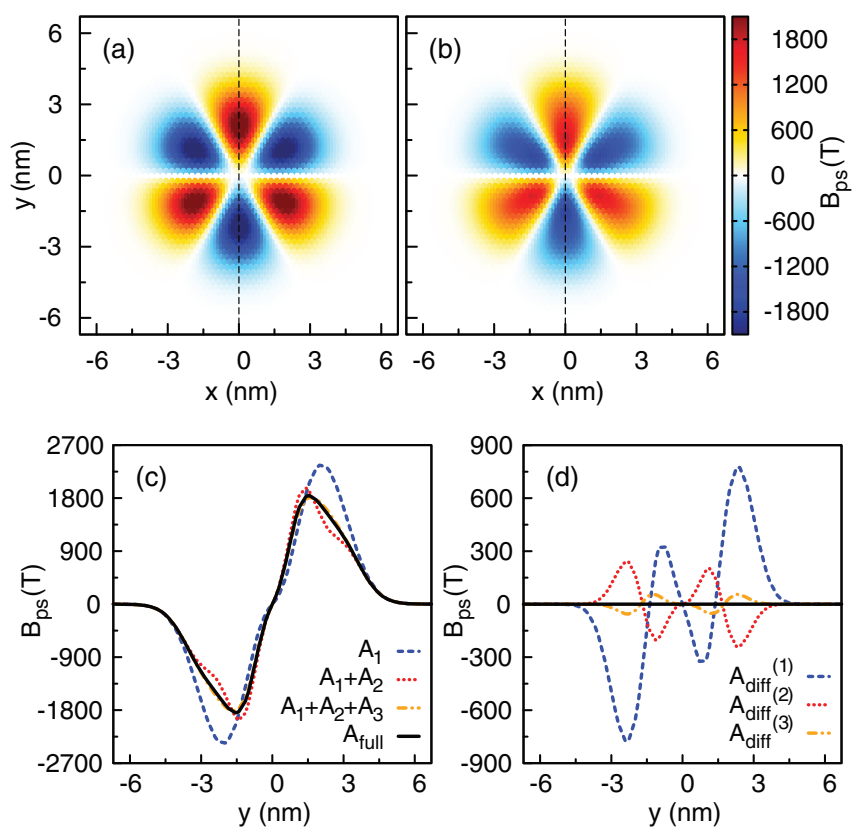

FIG. 3. (Color online) Top: Contour plots of the pseudomagnetic field generated by a Gaussian bump. The field is calculated (a) using the $A_{1}$ approximation of Eq. (7) and (b) using the full form of the vector potential Eq. (4). The dashed lines show cuts at $x=0$, along the armchair direction of graphene. Bottom: (c) Plot of the field calculated using successively higher order terms of the vector potential approximation $\left(A_{1}, A_{2}\right.$, and $\left.A_{3}\right)$ as well as the full form $A_{\text {full }}$ from Eq. (4). (d) The difference between the approximations and full solution as $A_{\text {diff }}^{(i)}=A_{i}-A_{\text {full }}$. In all cases the height of the bump is $h_{0}=2.2 \mathrm{~nm}$, which corresponds to a peak strain of $20 \%$.
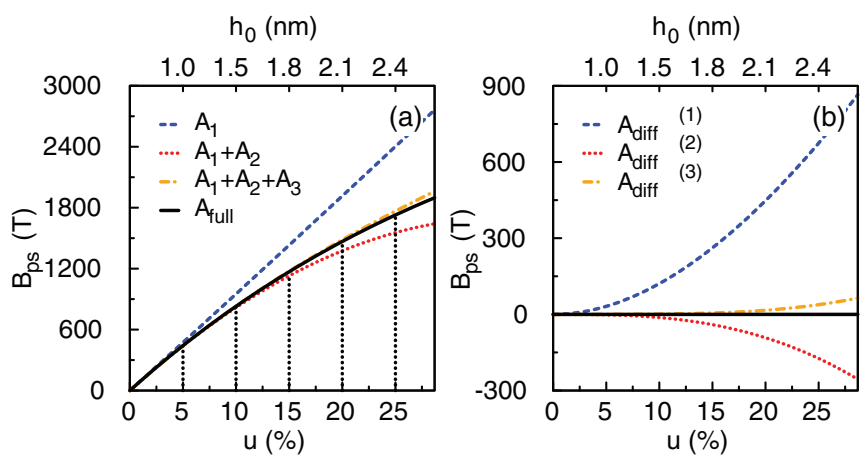

FIG. 4. (Color online) (a) Pseudomagnetic field at the location of maximum strain along the cut $(x=0, y=2.2 \mathrm{~nm})$. (b) The difference between the approximations and full solution as $A_{\text {diff }}^{(i)}=A_{i}-A_{\text {full }}$. The bump height $h_{0}$ is increased from 0 to $2.5 \mathrm{~nm}$, as indicated on the top $x$ axis, which generates the strain shown on the bottom $x$ axis.

the magnitude by as much as $800 \mathrm{~T}$. Adding the second order corrections $\left(A_{2}\right)$ will give better agreement, but there are still large deviations in the region around $y=2.2 \mathrm{~nm}$ where the strain is maximum, as well as near the center of the bump. Finally, including the third order term $A_{3}$ will result in generally good agreement.

In order to better evaluate the accuracy of the different vector potential approximations as a function of the strain, we plot the field at a fixed point while changing the bump height. As can be seen in Figs. 4(a) and 4(b), approximation $A_{1}$ diverges from the full solution at values as low as $5 \%$ strain. Adding $A_{2}$, we find good agreement up to about $15 \%$, after which the field is increasingly underestimated. Finally, adding term $A_{3}$ yields good agreement up to $25 \%$ strain.

These results bring up two issues. First, even the second order term $A_{2}$ is not enough to sufficiently approximate the pseudomagnetic field for strain above $15 \%$. Expanding the approximation to third order would improve results, but that would just needlessly complicate matters. Second, even if the second order term were sufficiently accurate, its form is too complicated for analytical results. On the other hand, using the numerical approach, there is no need for this, as the full vector potential Eq. (4) can easily be calculated. Thus, we find that numerical methods are best suited for correctly calculate the pseudomagnetic field at graphene's high tolerance of up to $25 \%$ strain.

\section{THE ELECTRONIC STATES}

We derive the energy levels $E_{n}$ and wave functions $\Psi(x, y)$ of the bump strained graphene flake using the tight-binding Hamiltonian Eq. (1) with the effect of strain included via the modulation of the hopping parameter given by Eq. (2). We shall compare the results from the tight-binding approach with the pseudomagnetic field model from the previous section.

The local density of states (LDOS) is given by

$$
D(E, x, y)=\sum_{n}|\Psi(x, y)|^{2} \delta\left(E-E_{n}\right) .
$$

To calculate the LDOS numerically we introduce a Gaussian broadening,

$$
\delta\left(E-E_{n}\right) \rightarrow \frac{1}{\Gamma \sqrt{\pi}} \exp \left[-\frac{\left(E-E_{n}\right)^{2}}{\Gamma^{2}}\right] .
$$



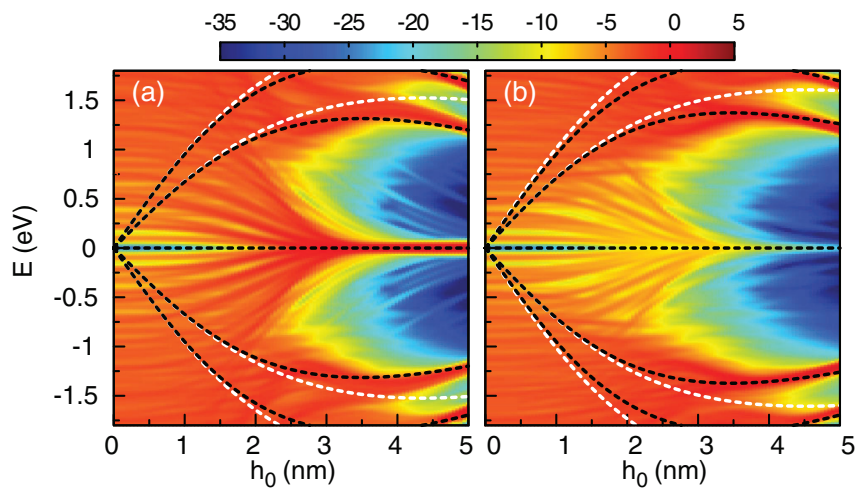

FIG. 5. (Color online) Contour plot of the LDOS for sublattice (a) $A$ and (b) $B$ as a function of bump height and energy, at the location of maximum strain ( $x=0, y=2.2 \mathrm{~nm}$ ). The dashed curves are the Landau levels based on the pseudomagnetic field model, calculated using unstrained (white curves) and strained (black curves) Fermi velocity.

As we did previously in Fig. 4, we select the location of highest strain along the armchair direction and we calculate the LDOS at that point in space as a function of bump height and energy. The results are shown in Fig. 5. At large bump heights, the LDOS shows Landau levels up to the second. Sublattice $B$ has a lower LDOS at the zero Landau level, which is consistent with what is found for a magnetic field in graphene.

For comparison, we calculate the Landau levels using the pseudomagnetic field model from the previous section and we overlay them on the LDOS as dashed curves in Fig. 5. In this case the pseudomagnetic field is calculated according to the full vector potential Eq. (4). Note that the Landau levels do not follow the usual square root function. This is because the plot is a function of bump height. The Landau levels still behave as a square root of the pseudomagnetic field.

In the first case (white dashed curves), the Landau levels are plotted for a constant unstrained Fermi velocity $v_{F}=$ $3 a_{c c} t_{0} / 2 \hbar$. This does not give good agreement with the LDOS when the bump height is large. In the second case (black dashed curves), the Landau levels are fitted to the LDOS with an adjusted strained Fermi velocity $v_{F}^{(s)}=3\left(a_{c c} t_{0}+\alpha l_{m n} \delta t_{m n}\right) / 2 \hbar$, where $\delta t_{m n}=t_{m n}-t_{0}$, and $\alpha=0.28$ is a fitting constant.

Next, we are interested in finding the spatial localization of the different electron states. We plot the energy levels as a function of bump height in Fig. 6. The levels split into two groups: Some energy levels decrease toward zero as the height of the bump increases, while the other group has the opposite behavior and increases slowly in energy with $h_{0}$. To better understand these two types of levels, we examine their wave functions. A point on a rising energy level is marked as $H$ in Fig. 6. The spatial probability for this state (see the right panel in Fig. 6) shows an electron state localized away from the center of the system, i.e., it is localized outside the bump. As the height of the bump is increased, the confinement area between the bump and the system edge is reduced. This reduction in confinement area results in an increase of the energy.

On the other hand, we have point $L$ which marks the level that splits downward, away from level $H$. The spatial probability in point $L$ is plotted in the right bottom panel of Fig. 6. These decreasing levels are confined inside the
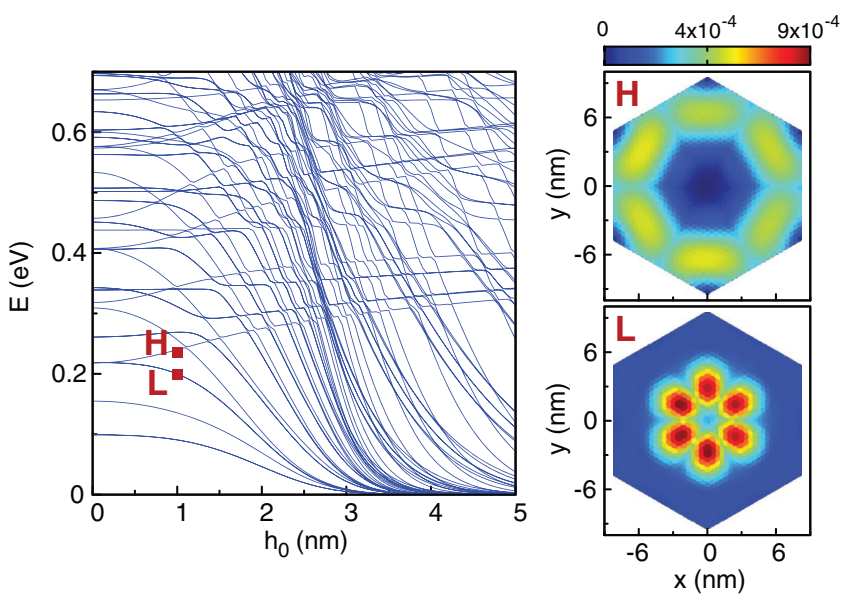

FIG. 6. (Color online) Energy spectrum of a hexagonal armchair graphene flake strained by a Gaussian bump. Left: Energy levels as a function of bump height. Right: Spatial probability at the split levels marked with $L$ and $H$.

bump, in contrast to the previous case. The probability peaks are found in the armchair directions which coincide with the peaks of the pseudomagnetic field from Fig. 3. These levels converge toward zero energy, thus forming the zero Landau level. Because the pseudomagnetic field is nonhomogeneous in this system, higher Landau levels are not clearly visible in the global energy spectrum.

The wave functions on the individual sublattices $A$ and $B$ are shown in Fig. 7 for both the $L$ and $H$ branches. Each sublattice contributes to three of the six probability peaks. The peak heights for each sublattice are the same, but the peaks are positioned opposite to one another. The areas of high probability for sublattice $A$ are positioned at the positive peaks of the pseudomagnetic field, while those localized on sublattice $B$ coincide with the negative peaks of the pseudomagnetic field. Note that for the $H$ level, the probability peaks are rotated by $60^{\circ}$ in the two sublattices as compared to the $L$ level. This also points to the different origin of these levels.

Another interesting feature of the energy levels are anticrossing points that switch the two types of energy levels. We

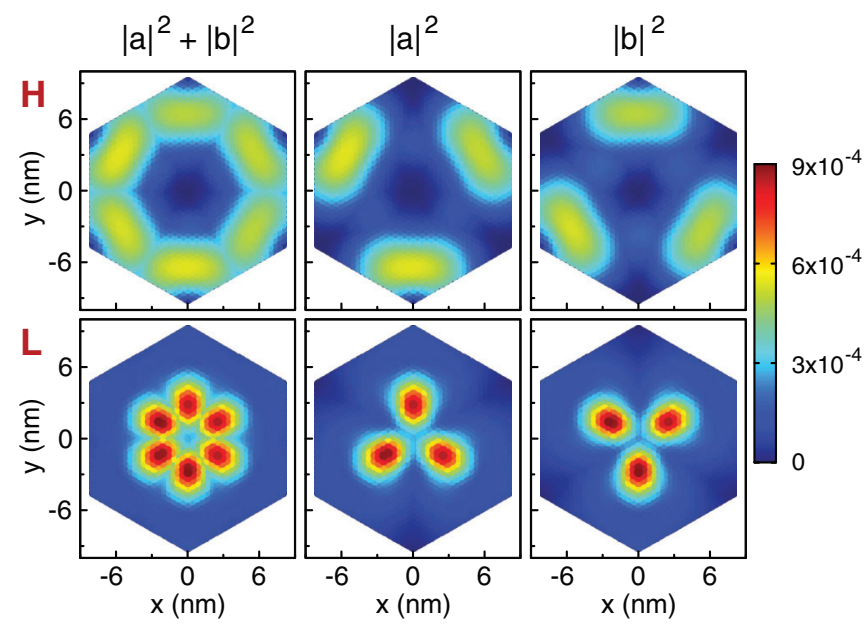

FIG. 7. (Color online) Electron probability for different sublattices at the points $L$ (bottom figures) and $H$ (top figures) from Fig. 6 . 

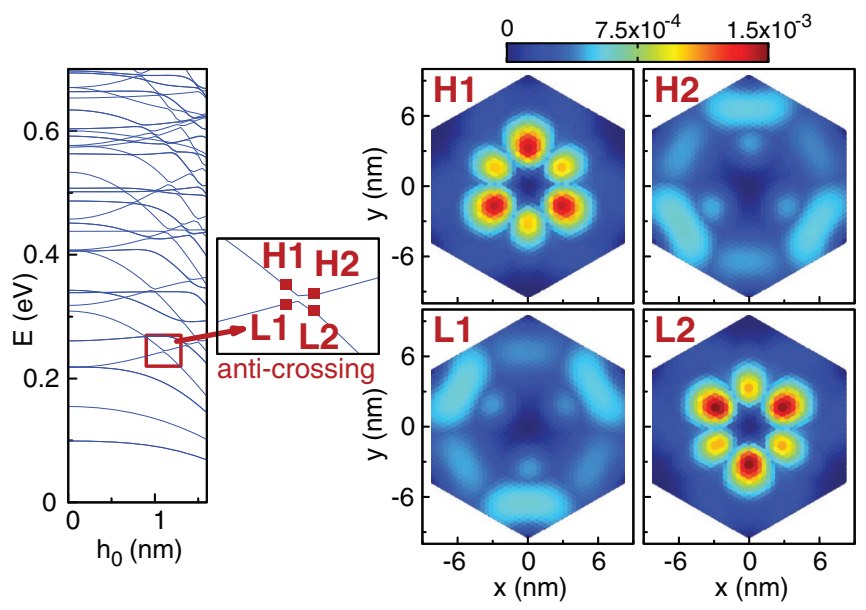

FIG. 8. (Color online) Energy levels as a functions of bump height in a hexagonal graphene flake with armchair termination. Left: Anticrossing point at bump height $h_{0}=1 \mathrm{~nm}$ and energy $E=0.24 \mathrm{eV}$. Right: Spatial probability before and after the anticrossing point.

examine one of these anticrossing points in detail in Fig. 8. We mark points $H 1, H 2(L 1, L 2)$ on the higher (lower) level before and after the anticrossing, respectively. Following the higher level from $H 1$ to $H 2$, we can see a transition from confinement inside the bump to confinement outside the bump. This is consistent with the previously discussed confinement types for decreasing and increasing energy levels with $h_{0}$. Following the lower level from $L 1$ to $L 2$ reveals the opposite behavior, with the confinement switching from outside to inside. Note also that when we go from $H 1$ ( $L 1)$ to $L 2$ ( $H 2)$ the positions of the peaks are rotated by $60^{\circ}$. The direction of the appearance of the peaks in the lower (higher) branch does not change when passing the anticrossing point.

The probability plots around the anticrossing point do not show perfect sixfold symmetry as we have seen in previous cases. Instead, we have two sets of three probability peaks with different magnitudes. We examine this asymmetry in Fig. 9 by plotting the separate probabilities for the two sublattices. At point $H 1$, sublattice $A$ has larger probability, but at point $H 2$ (after the anticrossing) this is reversed. Thus, following an

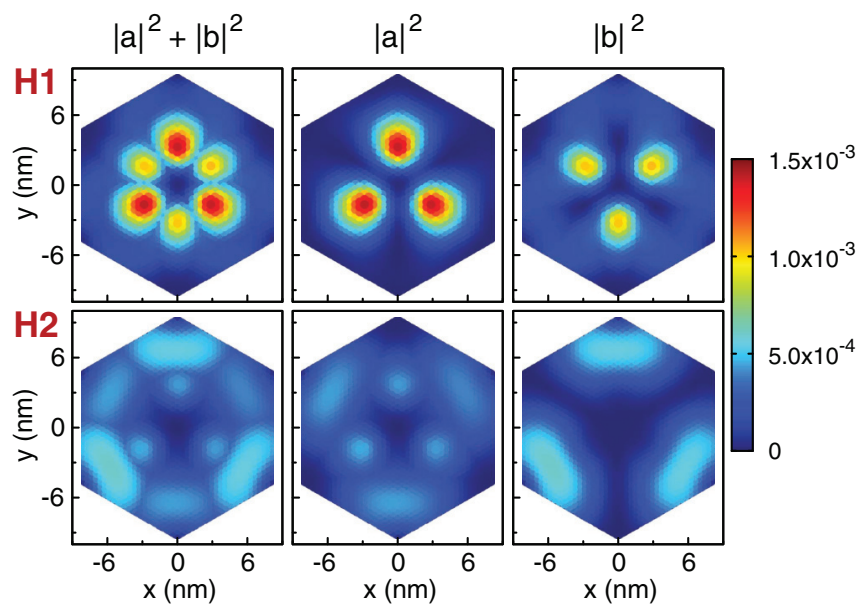

FIG. 9. (Color online) Electron probability for different sublattices before and after the anticrossing point from Fig. 8.

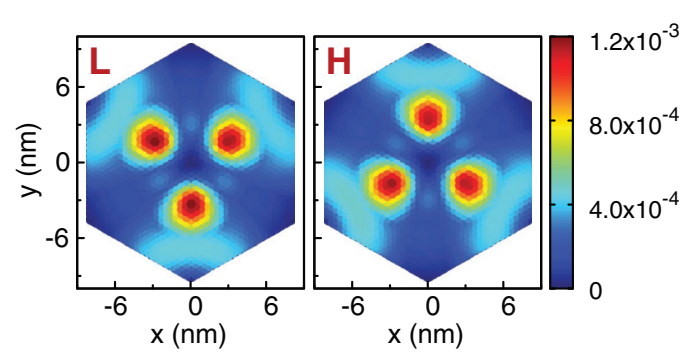

FIG. 10. (Color online) Electron probability at the lower $(L)$ and higher $(H)$ extrema of the anticrossing shown in Fig. 8.

energy level through an anticrossing point from $H 1$ to $H 2$ (or $L 1$ to $L 2$ ) will result in two transitions: Both the confinement type (inside or outside the bump) and the sublattice dominance are switched.

For completeness, we plot the probability distribution at the extrema of the anticrossing in Fig. 10. Notice that they exhibit an appreciable probability both inside and outside the bump. Both points are threefold symmetric but are rotated by $60^{\circ}$ relative to one another.

Since the bump is radial, it is natural to express the electron probability in polar coordinates as $P(r, \theta)$. We are specifically
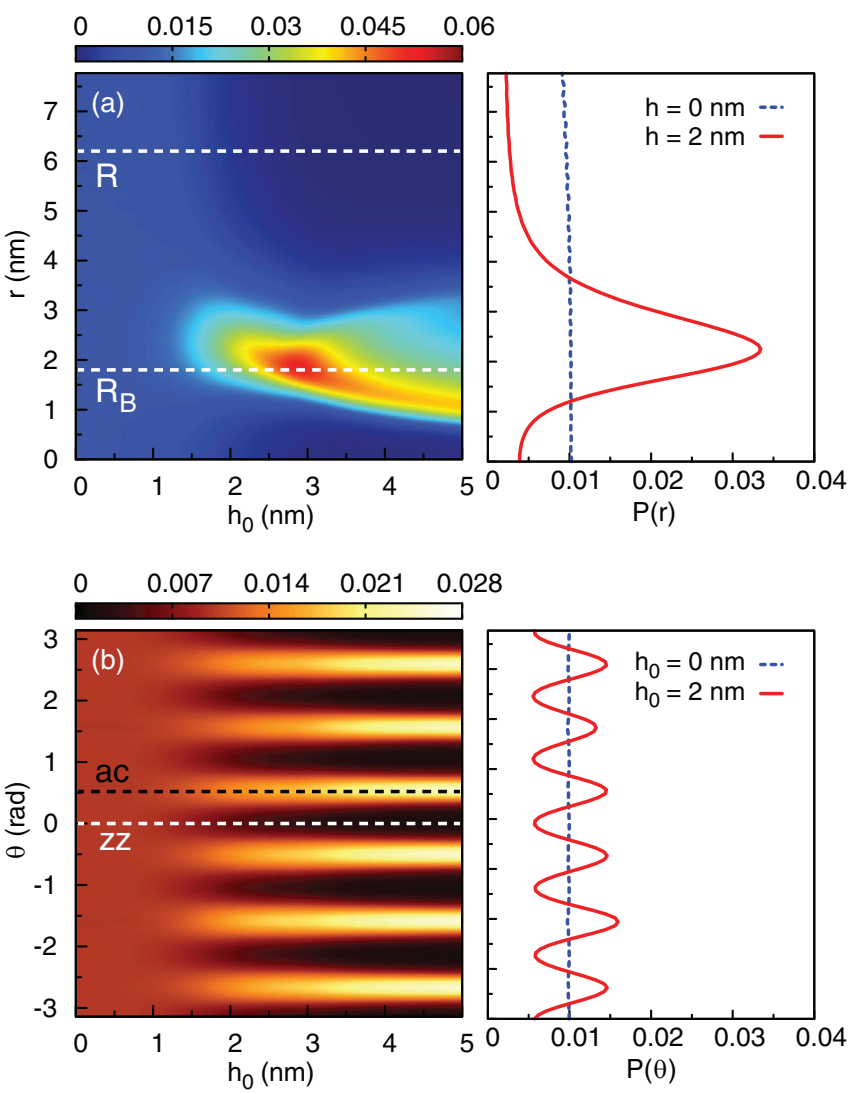

FIG. 11. (Color online) Left: (a) Radial and (b) angular electron probability at low energy as a function of the bump height. The dashed line labeled $R$ marks the radius of the bump and $R_{B}$ is the spatial position of the maximum of the pseudomagnetic field. In (b) the dashed lines indicate the armchair and zigzag directions in graphene. Right: Cuts of the probability at $h_{0}=0$ and $2 \mathrm{~nm}$. The energy is fixed at $E=0.05 \mathrm{eV}$. 
interested in finding electron states that are confined inside the bump and that are not influenced by the finite size of the simulated system. We can take an integral over the angle and only leave the radius dependent part of the probability

$$
P(r)=\int_{0}^{2 \pi} P(r, \theta) d \theta .
$$

Alternatively, we can do the opposite and take the integral over the radius, which leaves just the dependence on the angle.

The radial probability of low energy electrons as a function of bump height is shown in the left part of Fig. 11(a). As expected, the probability near zero bump height is practically uniform across the full radius of the system. As the height of the bump increases, we start seeing confinement inside the bump. More specifically, the probability peak is close to the position of the maximum of the pseudomagnetic field, marked as $R_{B}$.

Figure 11(b) shows the angular probability. The dashed lines indicate the armchair (black) and zigzag (white) directions of graphene, which alternate every $\pi / 6$ radians. For low bump height, the angular distribution is practically uniform. As the height of the Gaussian bump increases, probability maxima start to form in the armchair directions and minima appear in the zigzag directions.

Next, we fix the bump height at $h_{0}=2 \mathrm{~nm}$ and we present the probability as a function of energy in Fig. 12(a). For low
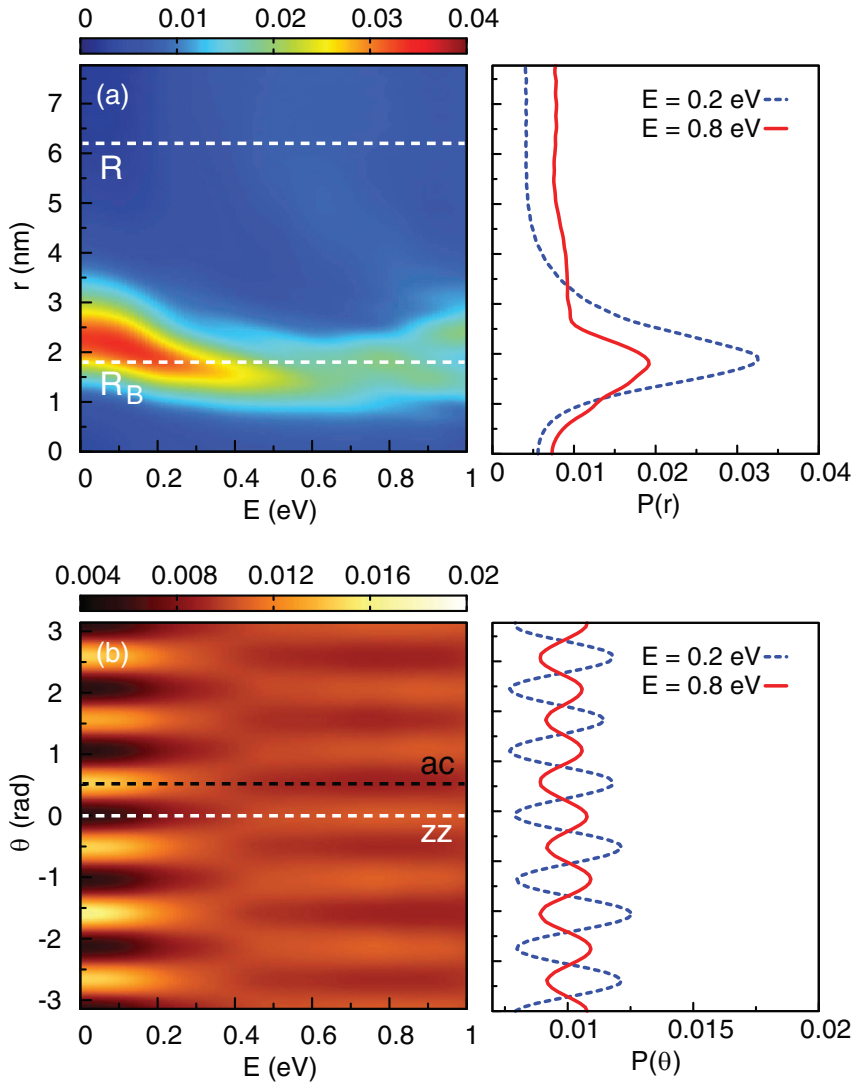

FIG. 12. (Color online) Left: (a) Radial and (b) angular electron probability as a function of energy. The marked lines are the same as in Fig. 11. Right: Cuts of the probability at $E=0.2$ and $0.8 \mathrm{eV}$. The bump height is fixed at $h_{0}=2 \mathrm{~nm}$. energy, we find that the state is mostly confined inside the bump near the strain maximum. But for energies above $0.3 \mathrm{eV}$, we find substantial probability outside the bump and thus weaker confinement.

Looking at the angular plot in Fig. 12(b), we find probability peaks in the armchair directions and minima in the zigzag directions. As the energy increases, the peaks disappear around $0.3 \mathrm{eV}$, which is the same energy where we started seeing substantial probability outside the bump in the radial plot. Once the probability outside the bump becomes substantial (above $0.4 \mathrm{eV}$ ), the highest probability shifts to the zigzag directions. As we will see later, the zigzag directions are associated with directions along which the probability current flows, connecting the center and the outside of the bump.

The single valley probability current at carbon atom $m$ is given by

$$
\vec{j}_{m}=\frac{i}{\hbar} \sum_{n=1}^{3} \Psi_{m}^{*} H_{m, m+n} \Psi_{m+n} \vec{d}_{n},
$$

where $H_{m, m+n}=-t_{m, m+n}$ is the tight-binding Hamiltonian matrix element. We plot the current inside the hexagonal flake in Fig. 13 for the same $L$ state as in Fig. 6. For clarity, the current is plotted separately for sublattices $A$ and $B$. Circular orbits coincide with the positions of the six probability peaks in the armchair directions. The current is very low at the exact positions of the probability peaks, but there is an appreciable circular current flowing around the peaks. Each sublattice contributes three circular orbits, where sublattice $A$ orbits have a clockwise direction and sublattice $B$ is counterclockwise. This coincides with the probability peaks of the individual sublattices, as well as with the positive and negative peaks of the pseudomagnetic field. Lines where the pseudomagnetic
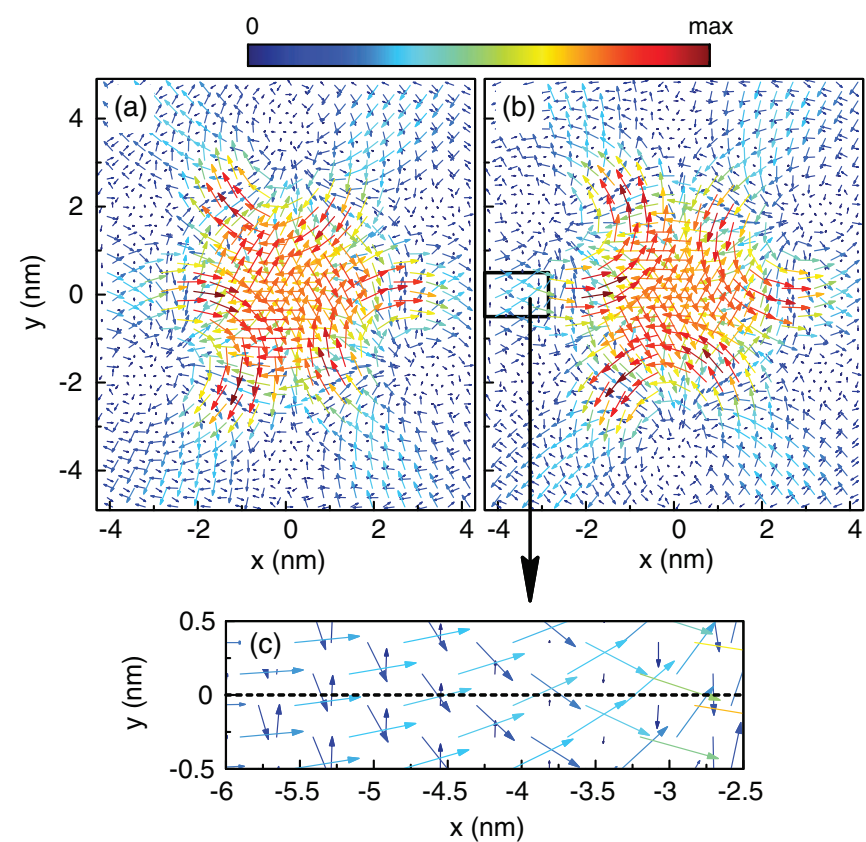

FIG. 13. (Color online) Probability current for the $L$ point electron state from Fig. 6, for sublattices (a) $A$ and (b) $B$. (c) An enlarged region around snake states. The dashed line indicates the zigzag direction. 
field is zero lie along the zigzag directions. Figure 13(c) shows the current along this line, where we find the current flowing successively across the line in both directions. These are snake orbits which are present because of the interface between the positive and negative pseudomagnetic field.

\section{CONCLUSIONS}

We showed that a circular symmetric straining of a hexagonal graphene flake induces a noncircular symmetric pseudomagnetic field. The average induced pseudomagnetic field is zero and the field changes sign when we cross a zigzag direction. The pseudomagnetic field was calculated up to third order in the strain. The first order term was found to be valid only up to $5 \%$ strain. The second order term extends the validity of the pseudomagnetic field expression for a Gaussian bump up to $15 \%$, while the third order is needed to go up to graphene's full strain limit $(25 \%)$.

Next, we investigated the confinement of electronic states in the same system. We found that nonuniform strain has a significantly different effect in the two fundamental directions of graphene. The sixfold symmetry of the confinement is directly related to the armchair and zigzag directions. Electrons are well confined in the armchair directions, while the zigzag directions allow the flow of probability current between the inside and outside of the strained region. This mirrors the form of the strain-generated pseudomagnetic field, which has peaks in the armchair direction and zero magnitude in the zigzag directions.

The energy levels of the Gaussian bump system show splitting and anticrossing states that correspond to different regions of localization of the electron. The levels that increase in energy with increasing bump height are confined between the bump and the edges of the graphene flake, while the decreasing levels correspond to states confined inside the bump. We also identified several anticrossing points which switch the confinement type (inside or outside the bump) as well as the sublattice dominance.

We examined the probability of finding the electron in the system as a function of the radius and angle. At low enyergy there is strong confinement inside the bump near the strain maximum. At higher energy, confinement is weaker as more states are found outside the bump. As for directional confinement, we found that low energy states are well confined in the armchair directions, where we see closed circular electron orbits. Higher energy states are more likely found in the zigzag directions, where the probability current shows channels to and from the center of the bump.

\section{ACKNOWLEDGMENTS}

This work was supported by the European Science Foundation (ESF) under the EUROCORES Program EuroGRAPHENE within the project CONGRAN, the Flemish Science Foundation (FWO-Vl), and the Methusalem Funding of the Flemish Government. *dean.moldovan@uantwerpen.be

†mrmphys@gmail.com

†francois.peeters@uantwerpen.be

${ }^{1}$ K. S. Novoselov, A. K. Geim, S. V. Morozov, D. Jiang, M. I. Katsnelson, I. V. Grigorieva, S. V. Dubonos, and A. A. Firsov, Nature (London) 438, 197 (2005).

${ }^{2}$ Y. Zheng, Y. W. Tan, H. L. Stormer, and P. Kim, Nature (London) 438, 201 (2005).

${ }^{3}$ Y. Zheng and T. Ando, Phys. Rev. B 65, 245420 (2002).

${ }^{4}$ F. Guinea, M. I. Katsnelson, and A. K. Geim, Nat. Phys. 6, 30 (2010).

${ }^{5}$ F. Guinea, A. K. Geim, M. I. Katsnelson, and K. S. Novoselov, Phys. Rev. B 81, 035408 (2010).

${ }^{6}$ A. H. Castro Neto, F. Guinea, N. M. R. Peres, K. S. Novoselov, and A. K. Geim, Rev. Mod. Phys. 81, 109 (2009).

${ }^{7}$ F. Guinea, M. I. Katsnelson, and M. A. H. Vozmediano, Phys. Rev. B 77, 075422 (2008).

${ }^{8}$ N. Levy, S. A. Burke, K. L. Meaker, M. Panlasigui, A. Zettl, F. Guinea, A. H. Castro Neto, and M. F. Crommie, Science 329, 544 (2010).

${ }^{9}$ V. M. Pereira and A. H. Castro Neto, Phys. Rev. Lett. 103, 046801 (2009).
${ }^{10}$ T. Georgiou, L. Britnell, P. Blake, R. V. Gorbachev, A. Gholinia, A. K. Geim, C. Casiraghi, and K. S. Novoselov, Appl. Phys. Lett. 99, 093103 (2011).

${ }^{11}$ H. Suzuura and T. Ando, Phys. Rev. B 65, 235412 (2002).

${ }^{12}$ A. L. Kitt, V. M. Pereira, A. K. Swan, and B. B. Goldberg, Phys. Rev. B 85, 115432 (2012).

${ }^{13}$ A. L. Kitt, V. M. Pereira, A. K. Swan, and B. B. Goldberg, Phys. Rev. B 87, 159909(E) (2013).

${ }^{14}$ F. de Juan, J. L. Manes, and M. A. H. Vozmediano, Phys. Rev. B 87, 165131 (2013).

${ }^{15}$ M. Ramezani Masir, D. Moldovan, and F. M. Peeters, Solid State Commun. (2013), doi: 10.1016/j.ssc.2013.04.001.

${ }^{16}$ C. Lee, X. Wei, J. W. Kysar, and J. Hone, Science 321, 385 (2008).

${ }^{17}$ K.-J. Kim, Ya. M. Blanter, and K.-H. Ahn, Phys. Rev. B 84, 081401(R) (2011).

${ }^{18}$ G. M. M. Wakker, R. P. Tiwari, and M. Blaauboer, Phys. Rev. B 84, 195427 (2011)

${ }^{19}$ V. M. Pereira, A. H. Castro Neto, and N. M. R. Peres, Phys. Rev. B 80, 045401 (2009).

${ }^{20}$ N. N. Klimov, S. Jung, S. Zhu, T. Li, C. A. Wright, S. D. Solares, D. B. Newell, N. B. Zhitenev, and J. A. Stroscio, Science 336, 1557 (2012). 